\title{
GenePainter: a fast tool for aligning gene structures of eukaryotic protein families, visualizing the alignments and mapping gene structures onto protein structures
}

\author{
Björn Hammesfahr ${ }^{1 \dagger}$, Florian Odronitz ${ }^{1+}$, Stefanie Mühlhausen ${ }^{1}$, Stephan Waack ${ }^{2}$ and Martin Kollmar ${ }^{1 *}$
}

\begin{abstract}
Background: All sequenced eukaryotic genomes have been shown to possess at least a few introns. This includes those unicellular organisms, which were previously suspected to be intron-less. Therefore, gene splicing must have been present at least in the last common ancestor of the eukaryotes. To explain the evolution of introns, basically two mutually exclusive concepts have been developed. The introns-early hypothesis says that already the very first protein-coding genes contained introns while the introns-late concept asserts that eukaryotic genes gained introns only after the emergence of the eukaryotic lineage. A very important aspect in this respect is the conservation of intron positions within homologous genes of different taxa.

Results: GenePainter is a standalone application for mapping gene structure information onto protein multiple sequence alignments. Based on the multiple sequence alignments the gene structures are aligned down to single nucleotides. GenePainter accounts for variable lengths in exons and introns, respects split codons at intron junctions and is able to handle sequencing and assembly errors, which are possible reasons for frame-shifts in exons and gaps in genome assemblies. Thus, even gene structures of considerably divergent proteins can properly be compared, as it is needed in phylogenetic analyses. Conserved intron positions can also be mapped to userprovided protein structures. For their visualization GenePainter provides scripts for the molecular graphics system PyMol.
\end{abstract}

Conclusions: GenePainter is a tool to analyse gene structure conservation providing various visualization options. A stable version of GenePainter for all operating systems as well as documentation and example data are available at http://www.motorprotein.de/genepainter.html.

Keywords: Exon, Intron, Gene structure, Evolution

\section{Background}

All eukaryotic genomes that have been sequenced so far have been shown to possess at least a few introns including the unicellular organisms that were previously suspected to be intron-less $[1,2]$. These data has fuelled the lively debate between the introns-early and intronslate concepts that is ongoing since the discovery of splicing [3]. The introns-early hypothesis says that already

\footnotetext{
* Correspondence: mako@nmr.mpibpc.mpg.de

${ }^{\dagger}$ Equal contributors

'Department of NMR-based Structural Biology, Max-Planck-Institute for Biophysical Chemistry, Am Fassberg 11, Göttingen 37077, Germany Full list of author information is available at the end of the article
}

the very first protein-coding genes contained introns while the introns-late concept asserts that eukaryotic genes gained introns only after the emergence of the eukaryotic lineage. Support for either of the concepts has been revealed by modelling the rates of intron gain and loss in eukaryotic genomes [4], by analysing the conservation of intron positions of example genes from a selection of genomes [5], or by population-genetic considerations [6]. Intron position conservation has also been used to improve gene predictions [7] and multiple protein sequence alignments [8], and to reconstruct ancient genes [9].

\section{() Biomed Central}

(C) 2013 Hammesfahr et al.; licensee BioMed Central Ltd. This is an Open Access article distributed under the terms of the Creative Commons Attribution License (http://creativecommons.org/licenses/by/2.0), which permits unrestricted use distribution, and reproduction in any medium, provided the original work is properly cited. 
A few software packages are available for the analysis of the conservation of intron positions. Exalign is a software using only gene structure information to reveal intron conservation [10]. Exalign uses gene structures from RefSeq gene annotations and from user definitions and calculates an alignment solely based on exon lengths and reading frames. Accordingly, Exalign fails if exon lengths between genes do not match. This is usually the case if genes encode less conserved proteins (e.g. differing in the lengths of surface loop regions) or if genes from more divergent species, which have been subject to complex intron loss and gain events, are compared. To overcome these limitations, it would be beneficial to use the information contained in protein sequence alignments. Tools that combine protein multiple sequence alignment (MSA) data and gene structures are CIDA/ CIWOG [11], GECA [12], Malin [13], and scripts developed for large-scale analyses [14,15]. CIDA/CIWOG comes with a web interface coupled to a database thus providing a barrier for installation while Malin requires a species phylogeny as starting point. GECA builds on the CIWOG output and provides the visualization of sequence similarity between subsequent genes. However, multiple sequence alignments are automatically generated and there is no option to use manually improved own alignments. In addition, sequence similarity is only computed for subsequent genes, which is inappropriate for large data sets. XdomView is the only software combining protein structures with intron and domain positions [16]. In XdomView the user specifies a PDB-ID and domain definitions from SCOP, CATH, DALI, 3DEE, and MMDB are subsequently mapped to the specified structure. In addition, the protein sequence from the PDB file is used to identify eukaryotic homologs in the ExInt database to map intron positions and phase. However, XdomView is strongly limited by accepting only PDB codes as input and the reference databases are far out of date (PDB of June 2003, SCOP release of March 2003, ExInt based on Genbank 122 of February 2001).

With GenePainter we developed a tool that combines protein MSA data, gene and protein structures. GenePainter maps the intron positions obtained from the gene structures to the MSA taking reading frames into account. Additionally, conserved intron positions can be displayed in provided protein structures. The output can be used to compare gene structures from the exon/intron level down to the nucleotide sequences and to resolve and improve potentially ambiguous regions in the MSAs. GenePainter does not require any additional software/database to be installed and is unique compared to previous tools in its output options and the possible application in small- as well as larges-scale analyses.

\section{Implementation}

GenePainter was written in Ruby, does not require any additional library, and can be used on any operating system (Additional file 1). As input, GenePainter requires a protein MSA in FASTA format and corresponding gene structures in YAML format, which can be obtained for example from Scipio [17] or via the WebScipio interface $[18,19]$. User-specified options control the part of the alignment used in the comparison and the type of output. In addition, a PDB file can be provided to map gene structure conservation onto a protein structure. GenePainter starts with comparing sequence and gene structure file names. After processing the gene structure files, intron positions including phase information (phase 0,1 or 2 depending on the intron's position relative to the reading frame) and intron sequences are mapped to each sequence in the MSA. Introns are then grouped into clusters based on identical alignment positions and matched to each other. As matched introns are aligned, exons are filled with the respective placeholder for the text-based output. In the graphical output, exons are represented with their respective length, but stretched by placeholders if needed.

The text and SVG output can be processed with any appropriate software. The output to visualize intron positions mapped to structures is scripts for PyMOL [20]. The software as well as a comprehensive documentation can be found at http://www.motorprotein.de/genepainter.html.

\section{Needleman-Wunsch}

The mapping of intron positions and phases onto a PDB file is based on an alignment of the PDB sequence with one of the sequences from the protein MSA as reference. Thus, both the reference sequence and the chain of interest from the PDB file need to be specified. The alignment is calculated as described in [21]. By default, gaps at the end of the alignment are not penalized. This adaptation is of particular importance, as reference and protein sequence may vary greatly in length, possibly leading to an inappropriate alignment. Reasons for length differences can be full-length sequence in the alignment versus sequence of a single domain in the crystal structure, protein sequence in the alignment versus sequence joined to an expression/purification tag in the structure, and missing parts in the structure due to missing electron density.

\section{Results and discussion}

To demonstrate GenePainter we use part of the coronin dataset published recently [22]. For test and evaluation purposes GenePainter has also been applied to other protein families with different numbers of genes, introns per gene, and MSA lengths (Additional file 2). Coronins are a family of actin remodelling proteins consisting of a 


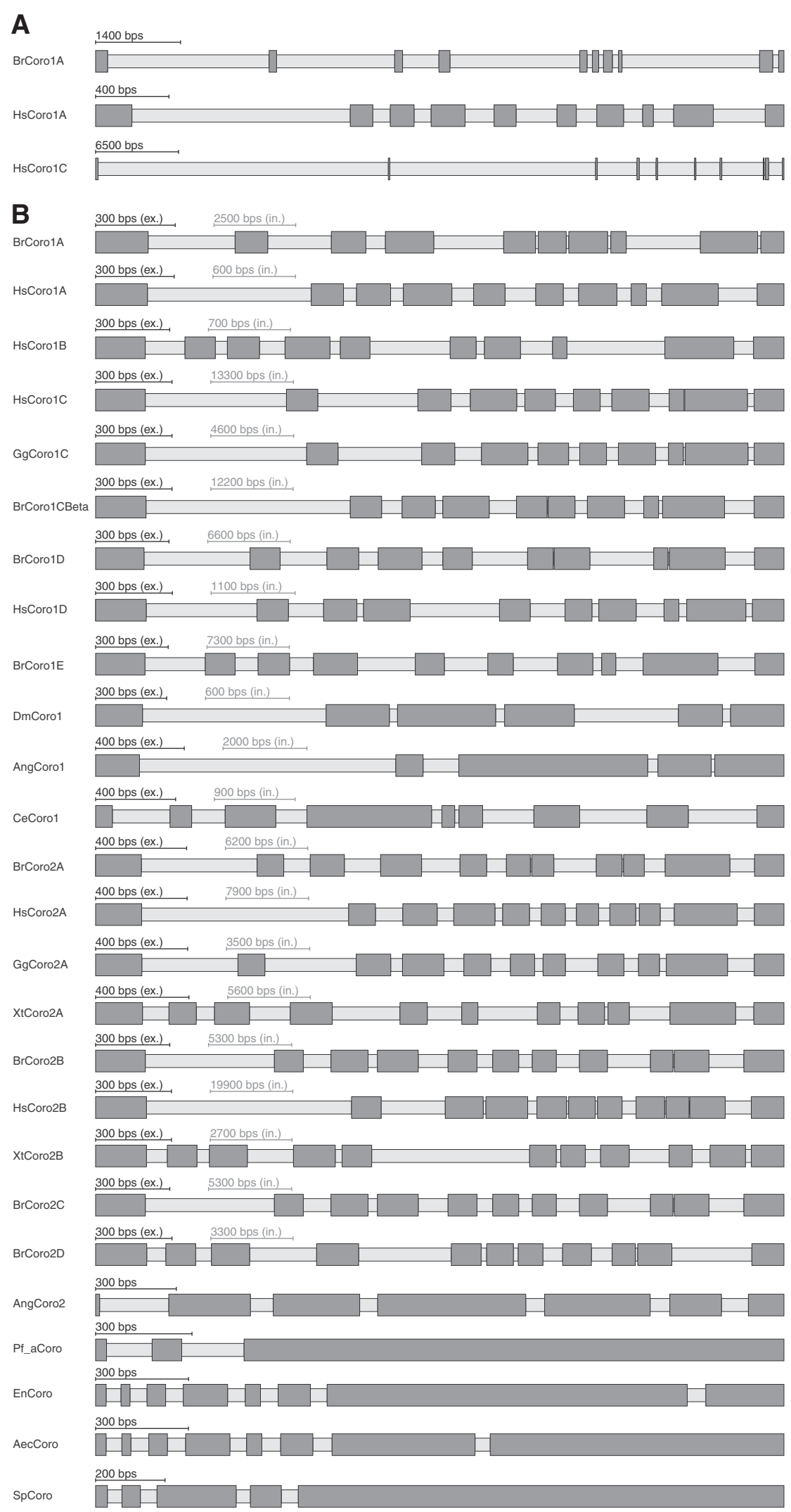

Figure 1 (See legend on next page.) 

zebrafish ( $\mathrm{Br}=$ Brachydanio rerio) Dark grey bars and light grey bars mark exons and introns, respectively. B) This figure illustrates examples of coronin genes from vertebrates ( $\mathrm{Hs}=$ Homo sapiens, $\mathrm{Br}=$ Brachydanio rerio, $\mathrm{Gg}=$ Gallus gallus, $\mathrm{Xt}=$ Xenopus tropicalis), arthropods

$(\mathrm{Dm}=$ Drosophila melanogaster, $\mathrm{Ang}=$ Anopheles gambiae $)$, nematods $(\mathrm{Ce}=$ Caenorhabditis elegans $)$ and the protozoan parasite Plasmodium falciparum (Pf_a). In order not to make small exons vanish when very large intronic stretches are present, the scaling of introns and exons is automatically balanced to make the picture visually meaningful (scale bars for exons and introns are given, respectively). Here, except for AngCoro2 and Pf_aCoro in all schemes the introns were scaled down and the exons scaled up so that the average length of the introns equals the average length of the exons.

conserved $\beta$-propeller domain, that comprises the $\mathrm{N}$ terminal two thirds of the sequences, a unique region, which is only conserved within closely related species, and a short $\mathrm{C}$-terminal coiled-coil region that mediates trimerization. Also, a protein structure is available comprising the $\beta$-propeller domain and part of the unique region [23]. GenePainter needs FASTA formatted protein MSAs and gene structure information stored in YAML files as input (Figure 1, Additional file 1). Optionally, a protein structure can be provided in PDB format. The gene structures can most easily be obtained by using the WebScipio $[18,19]$ web interface or via the WebScipio web service by using the provided gene_scan.rb script. The latter option requires the user to specify species names and genome assemblies, which are easier to select via the WebScipio web interface. The advantage of using Webscipio is its ability to predict protein sequences and reconstruct gene structures in cross-species searches [19] and thus the possibility to easily extend the input data by adding genes from related species. Also, Webscipio can cope with genome assembly problems like assembly gaps and sequencing errors leading to frame shifts and in-frame stop codons in exons. In the current implementation, other file formats describing gene structures like GFF [24] cannot be used as alternative input files for GenePainter. This is due to the fact that GFF files normally do not contain DNA sequence and therefore do not provide all necessary information. Optionally, alignment limits can be defined in GenePainter. This is particularly useful when comparing specific regions and domains of multi-domain proteins separately.

Because GenePainter compares gene structures based on multiple sequence alignments of proteins it can be used to analyse proteins of any degree of similarity. The coronins from the sample data comprise sequences from apicomplexans, fungi and mammals. Accordingly, the similarity of the gene structures is not obvious at first glance (Figure 1A). By scaling the exons and introns the similarity of exon lengths between homologs of closely related organisms becomes suggestive (Figure 1B. Note the different scaling of exons and introns in this figure). Exons and introns were scaled up and down, respectively, so that the average length of the exons equals the average length of the introns. GenePainter maps intron positions including phase to the sequences as provided in the multiple sequence alignments.

\section{Gene structure alignments}

The aligned gene structures can be analysed in various formats. The basic output format displays common intron positions in plain text, where introns are represented by vertical bars "|" (Figure 2A). Hyphen-minuses "-" are used as spacers for better orientation and represent exonic sequence in abstract form. Optionally, the hyphen-minuses can be replaced by spaces so that the output just represents common introns (Figure 2B). These formats are particularly useful for visualizing large-scale data (many positions and sequences in the MSAs) and are independent of exon and intron lengths. Additional information can be added by using the $-n$ option of GenePainter by which intron positions are represented by phase numbers instead of vertical bars "|" (Figure $2 \mathrm{C}$ ). With the option -phylo the common intron data is transformed into an intron present " 1 " - intron absent " 0 " format, which can be used to calculate phylogenetic trees based on gene structure data (Figure 3).

A gene structure alignment including exon and intron lengths is shown in Figure 4. The same alignment is drawn with varying degrees of detail (Figure 4A, 4B). In the most reduced picture the focus lies on common introns (Figure 4A). The intron length is not included in this figure, but in Figure $4 \mathrm{~B}$, which provides most information about the underlying gene structures. Here, it becomes immediately obvious that intron lengths vary considerably (compare human $\mathrm{HsCoro} 2 \mathrm{~B}$ and frog $X t$ Coro2B for example). In addition, the scheme shows that the $\mathrm{N}$-terminal $\beta$-propeller domain of coronin is highly conserved while the unique regions are variable and contain many gaps in the multiple sequence alignment (blue bars; Figure 4B).

The gene structure information can also be incorporated into the protein MSA as additional lines (option -a) where intron positions are either displayed as vertical bars "|" or as numbers defining the phase of the respective introns (Figure 4C). This format is most useful if the MSA will be re-evaluated to identify miss-aligned positions and regions. 
A

$>$ BrCorolA $>$ HsCorolA $>$ HsCorolB

$>$ HsCorolC

$>$ GgCorolC

$>$ BrCorol Cbeta

$>$ BrCorolD

$>$ HsCoro1D

$>$ BrCorolE

$>$ DmCoro1

$>$ AngCorol

$>$ CeCoro1

$>$ BrCoro2A

$>$ HsCoro2A

$>$ GgCoro2A

$>\mathrm{XtCorO} 2 \mathrm{~A}$

$>$ BrCoro2B

$>$ HsCoro2B

$>\mathrm{XtCor} 2 \mathrm{~B}$

$>$ BrCoro2 $\mathrm{C}$

$>$ BrCoro2D

$>$ AngCoro2

$>$ Pf_aCoro

$>$ EnCoro

$>$ AecCoro

$>$ SpCoro

B

$>$ BrCorolA

$>$ HsCorolA

$>$ HsCorolB

$>$ HsCorolC

$>$ GgCorolC

$>$ BrCorolCbeta

$>$ BrCorolD

$>$ HsCorolD

$>$ BrCorolE

$>$ DmCorol

$>$ AngCorol

$>$ CeCorol

$>$ BrCoro2A

$>$ HsCoro2A

$>$ GgCoro2A

$>$ XtCoro2A

$>$ BrCoro2B

$>$ HsCoro2B

$>$ XtCoro2B

$>$ BrCoro $2 \mathrm{C}$

$>$ BrCoro2D

$>$ AngCoro 2

$>$ Pf_aCoro

$>$ EnCoro

$>$ AecCoro

$>$ SpCoro

C

$>$ BrCorolA

$>$ HsCorolA

$>$ HsCorolB

$>$ HsCorolC

$>$ GgCorolC

$>$ BrCorolCbeta

$>$ BrCorolD

$>$ HsCorolD

$>$ BrCorolE

$>$ DmCorol

$>$ AngCorol

$>$ CeCoro1

$>$ BrCoro2A

$>$ HsCoro2A

$>$ GgCoro2A

$>\mathrm{XtCoro} 2 \mathrm{~A}$

$>$ BrCoro2B

$>$ HsCoro2B

$>$ XtCoro2B

$>$ BrCoro2 C

$>$ BrCoro2D

$>$ AngCoro2

$>$ Pf_aCoro

$>$ EnCoro

$>$ AecCoro

$>$ SpCoro
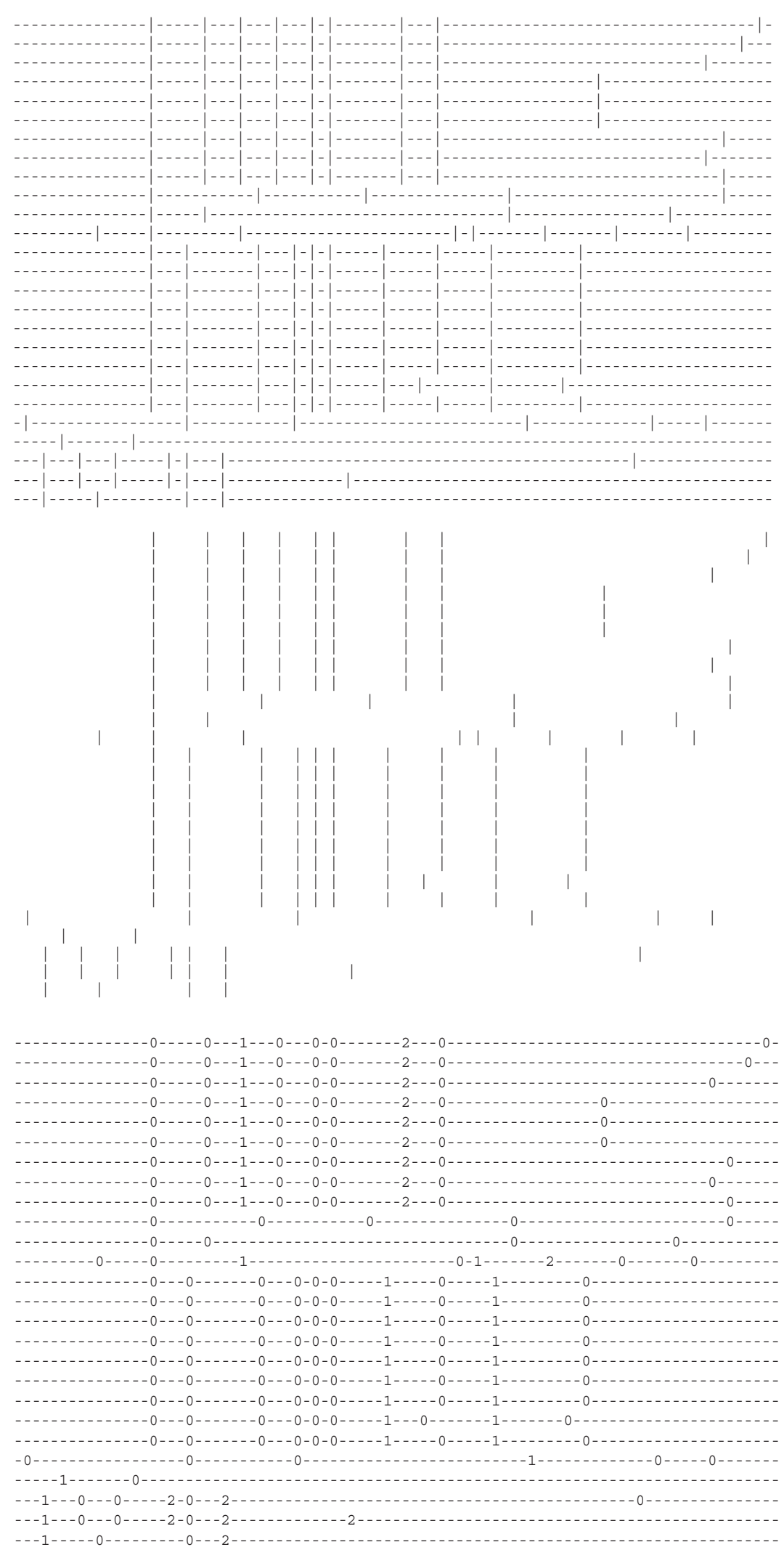

Figure $\mathbf{2}$ (See legend on next page.) 


\section{(See figure on previous page.)}

Figure 2 Gene structure alignments. The gene structures shown in Figure 1 were aligned with GenePainter. Three visualization options focusing on common introns exist. In each, exons and introns are represented independent of their length. A) In this gene structure alignment, coding sequences are represented by "-" and introns by "|". "|" underneath each other indicate intron positions with the same phase at the same position in the multiple sequence alignment. B) Here, only introns are pictured. Coding sequences are denoted by spaces, introns by "I". C) Similar visualization as in $\mathbf{A}$ ) except that the intron phases are given instead of the intron indicator "|". All outputs shown are plain text files and can be analysed with any text editor.

\section{Visualizing conserved intron positions on protein structures}

Gene structure conservation derived by GenePainter can further be mapped on protein structures (option $-\mathrm{pdb}$ <file> [chain]). Therefore, one of the proteins from the MSA (set by -pdb_prot) is taken as reference and aligned with the protein sequence from a PDB file.
Generating the alignment with standard NeedlemanWunsch parameters, which penalize gaps at the end of the alignment, can be forced by setting the option -penalize_endgaps. Based on this alignment, intron positions and phases are projected onto the protein structure. If reference gene and protein structure chain are not specified, the first sequence in the alignment

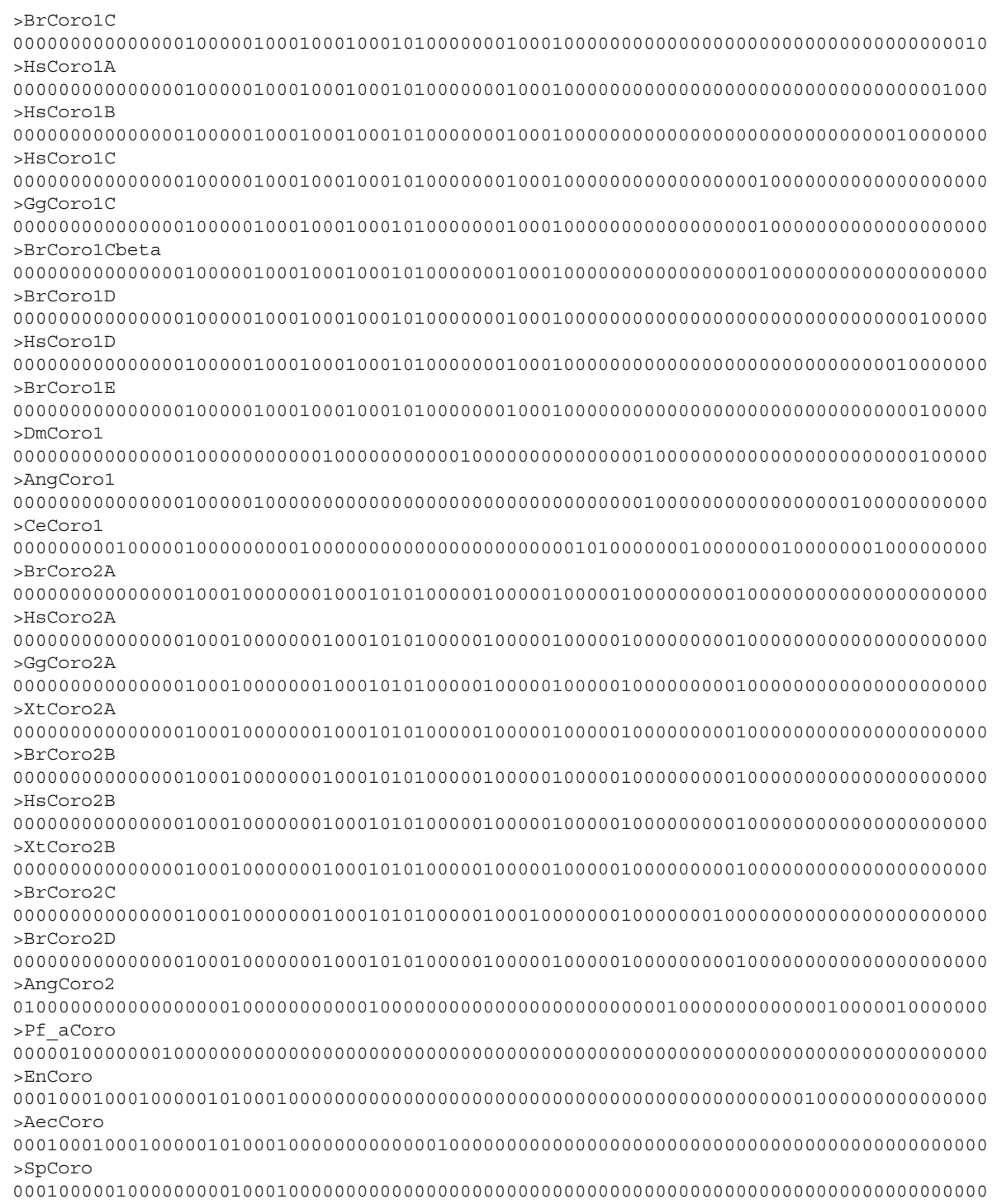

Figure 3 Binary representation of gene structures. The binary representation of aligned intron positions is in FASTA format. The presence and absence of common introns are denoted by ones and zeros, respectively. 
A

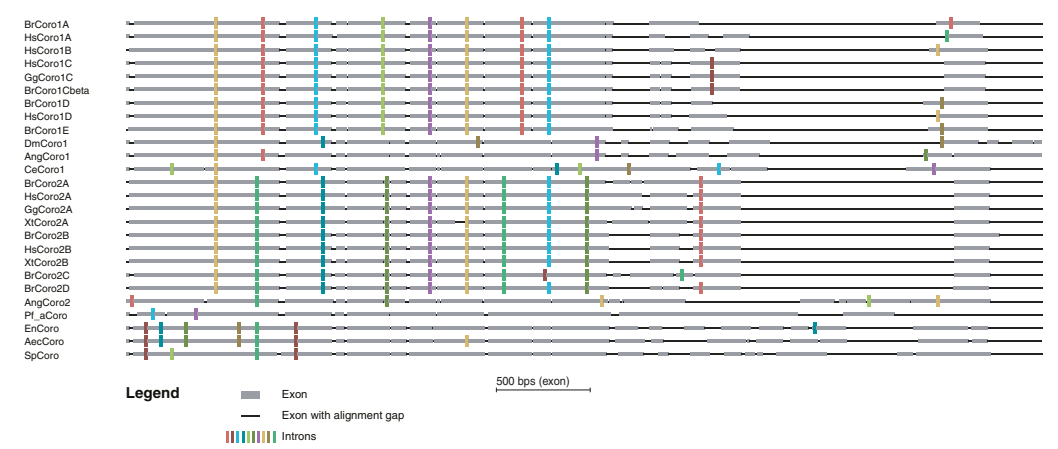

B

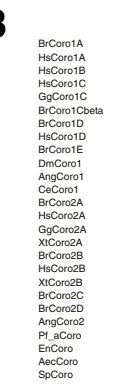
C EnCoro
AecCoro
SpCoro
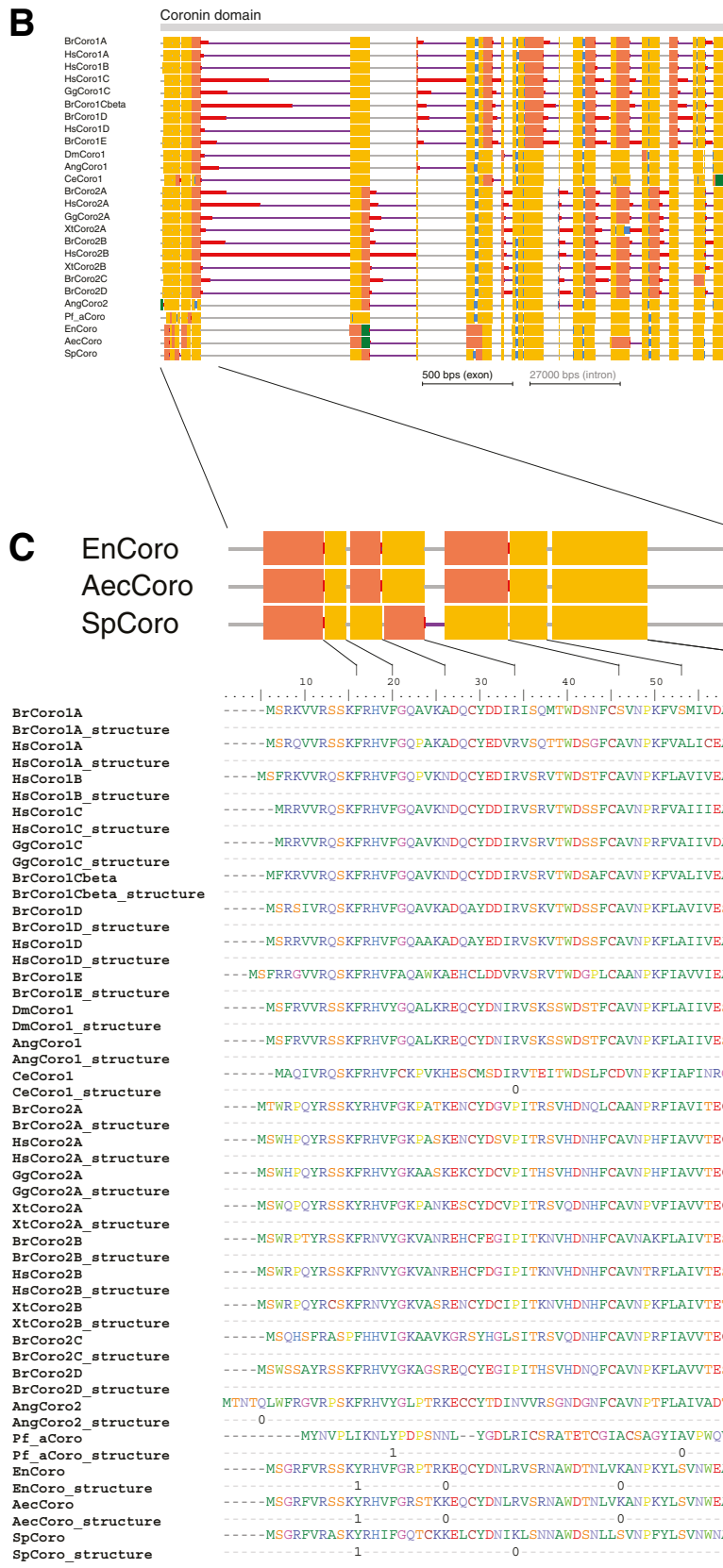

Unique region CoiledCoil
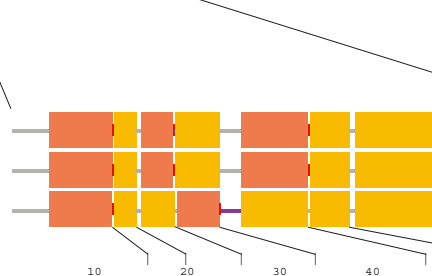

Exon not interrupted by any feature in other sequences

Exonic region interrupted by features in other sequences

Exon placeholder

Last part of exonic region before an intron

- Exon with missing DNA sequence

- Intron
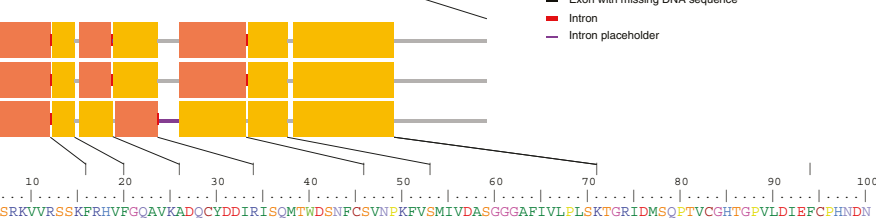
作 MSRQVVRS SKFRHVFGQPAKADQCYEDVRVSQTTWDSGFCAVNPKFVALICEASGGGAFLVL LGKTGRVDKNA TVCGHTA VVDIAWCPHNDN MSFRKVVRQSKFRHVFGQ VKNDQCYEDIRVSRVTWDSTFCAVN KFLAVIVEASGGGAFLVL LSKTGRIDKAY TVCGHTG PVLDIDWCPHNDE -MRRVVRQSKFRHVFGQAVKNDQCYDDIRVSRVTWDSSFCAVN RFVAIIIEASGGGAFLVL LHKTGRIDKSY YVCGHTG VVLDIDWCPHNDQ -MRRVVRQSKFRHVFGQAVKNDQCYDDIRVSRVTWDSSFCAVN RFVAI IVDASGGGAFLVL LHKTGRIDKSY TVCGHTG VVLDIDWCPHNDQ MFKRVVRQSKFRHVFGQAVKNDQCYDDIRVSRVTWDSAFCAVVN KFVALIVEASGGGAFMVL PLHKTGRIDKAY PTVCGHTG VVLDIDWCPHNDQ MSRS IVRQSKFRHVFGQAVKADQAYDDIRVSKVTWDSSFCAVNN KFLAVIVESSGGGAFLVM LSKMGRVDKNY LVVGHSG VVLDIDWC HNDN -MSRRVVRQSKFRHVFGQAAKADQAYEDIRVSKVTWDSSFCAVNVKFLAIIVEAGGGGAFIVL LAKTGRVDKNY LVIGHTA VLDIDWCPHNDN MSFRRGVVR S SKRHVFAQANKAEHCLDDVRVSRVTWDG LCAANPKFIAVVI IAGGGGAFLVL LAKSGRVDQNTP TVCGHAA VVLDVQWC PHDDN -MS FRVVRS SKFRHVYGQALKREQCYDNIRVSKSSWDSTFCAVNPKFLAI IVESAGGGAFIVL HNKVGRIAADH LVGGHKG VLDIAWCPHNDN -MSFRVVRS SKFRHVFGQALKREQCYDNIRVSKSSWDSTFCAVN KFLAI IVESAGGGAFIVL HNKVGRIAADHALVGGHKG VVLDIAWCPHNDN -MAQIVRQSKFRHVFCK VKHESCMSDIRVTEITWDSLFCDVNPKFIAFINRGA-GG FMVI VVNKIGRVDKDY Y FVDAHKA CLEVANS FNDN MTWR QYRSSKYRHVFGK ATKENCYDGVITRSVHDNQLCAAN RFIAVITECAGGGSFLVL VHHTGKVD PHH KVSGHRGNVLDI KWNHFNDF MSWHP QYRSSKFRHVFGK ASKENCYDSVP ITRSVHDNHFCAVNNHFIAVVTECAGGGAFLVI PLHQTGKLD PHY KVCGHRGNVLDVKWNPFDDF MSWH QYRS SKFRHVYGKAASKEKCYDCV ITHSVHDNHFCAVNPHFIAVVTECAGGGAFLVI IHQTGKLD PHY RICGHKGNVLDIKWNP FNDF MSWQ QYRSSKYRHVFGK ANKE SCYDCVI ITRSVQDNHFCAVNPVFIAVVTECAGGGAFIVI L $Q Q S G K L D$ PHH KVCGHRGNVLDIKWND FDDY MSWR TYRSSKFRNVYGKVANREHCFEGI ITKNVHDNHFCAVNAKFLAIVTESAGGGSFVI IVTQFGRID PHY KVCGHQGNVLDI KWNP FHDN MSWR QYRS SKFRNVYGKVANREHCFDGI I ITKNVHDNFFCAVNTRFLAIVTESAGGGS LLVI LEQTGRIE PNY KVCGHQGNVLDIKWNPFIDN MSWR PYRCSKFRNVYGKVASRENCYDCI ITKNVHDNHFCAVNP KFLAIVTETAGGGSFLVI L QQTGRIE PNY KVCGHQGTVLDI KWNP FIEN -MSQHSFRAS P FHHVI GKAAVKGRS YHGLSITRSVQDNHFCAVNPRFIAVVTECTGGGAFIVISVRHTGRVS PLHARVCGHSARVLDVKWD FNDL MSWSSAYRSSKFRHVYGKAGSREQCYEGI ITHSVHDNQFCAVN KFLAVVTESAGGGAFIVI LHK GRVD HY RVCGHQGSVMDI KWS PFMDN TINTQLWFRGVR SKFRHVYGL TRKECCYTDINVVRSGNDGNFCAVNPTFLAIVADTT - - - FVVI IATTGRIDFQCCKVIGHAGQILDLKWNP FDDH 0-- MYNV LI KN Y D D SNNL- - YGDLRICSRATETCGIACSAGYIAVPWQVEGGMI GVIRLENOVRN - -VIKLKSHTS ILDLSFNPCYSE -MSGRFVRS SKYRHVFGR TRKEQCYDNLRVSRNAWDTNLVKANPKYLSVNWEAGGGGAFAVI PLERGKL ERI LFRGHTAVVLDTDWN FNDD MSGRFVRSSKYRHVFGRSTKKEQCYDNIRVSRNAWD TNLVKANP KYLSVNWEAGGGGAFAVI LEERGKL ERI L LFRGHTAVVLDTDWNP FNDD MS GRFVRASKYRH IFGQTCKKELCYDNI KLSNNAWD SNLLSVN FYLSVNWNAGAGGALAVI LNERGKL DQVNLFRGHTAAVLDTDWNP FHDQ

Figure 4 (See legend on next page.) 


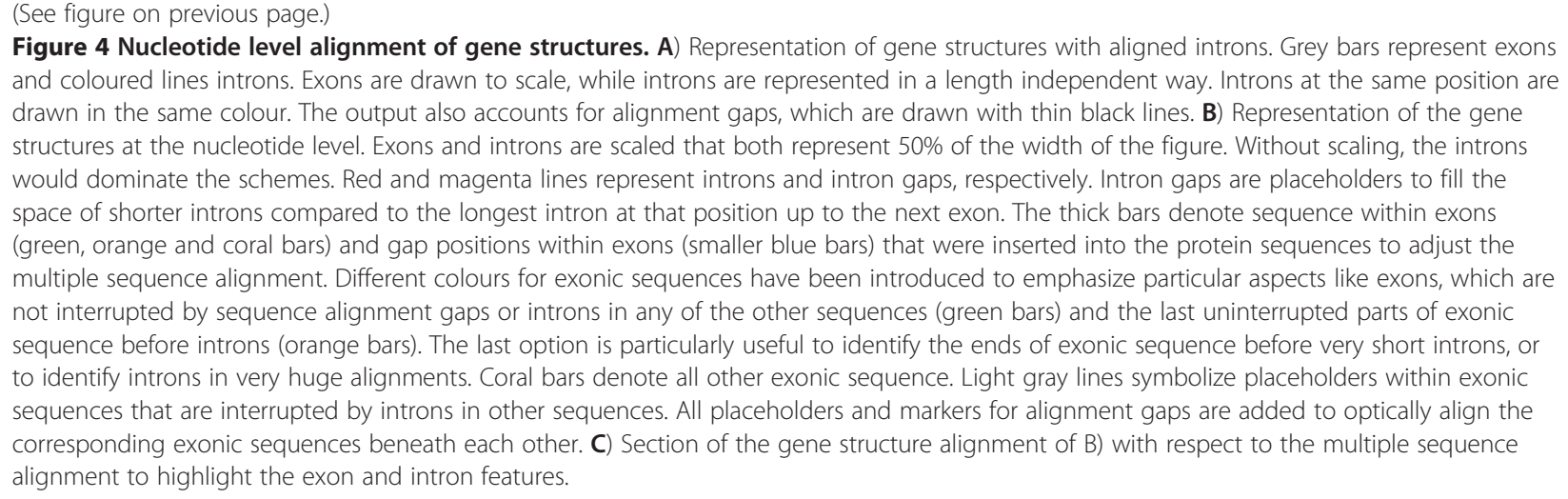

and chain A will be used by default. GenePainter generates two python scripts for execution within PyMol comprising all necessary steps (including loading the PDB) in order to display the mapped intron positions and phases. While the script color exons . py colours residues based on the underlying gene structure (Figure 5A), the other highlights only intron phases (color_splicesites.py; Figure $5 \mathrm{C}$ ). In this visualization, both the last and first residues of succeeding exons are coloured by a three-colour scheme denoting the phases of the respective introns. In order to elucidate the conservation of the respective intron positions, by default only those positions that are conserved in more than $80 \%$ of the genes of the alignment (parameter can be changed via-consensus) are considered for visualization (Figures 5B and 5D). In both visualizations attention is focused on those parts of the structure, on which intron data are mapped. Unused chains and regions not mapped to the reference sequence like cloning artefacts and protein purification tags are displayed in grey.

\section{Limits and possible applications of GenePainter}

In the current version, GenePainter is limited to group introns to common introns only if these appear at the exact same position and exact same reading frame in the aligned protein sequences. In contrast CIWOG defines introns, which happen in closely neighbouring amino acids, as common introns [11]. However, there are many examples of very short exons not only in mammals but also in other branches of the eukaryotes, which would unnecessarily be grouped as common introns. As we showed (Figure 4C) more and more conserved introns will appear in the alignment as soon as more sequences and gene structures are added. For instance, the Schizosaccharomyces pombe (SpCoro) and Caenorhabditis elegans (CeCoro1) coronin genes share an intron at the $\mathrm{N}$-terminus (Figure $4 \mathrm{C}$ ). We believe that almost all introns are shared between at least two genes from two different species. However, most analyses do not cover enough data, not enough sequences and not enough species. We have shown previously that all introns within the arthropod muscle myosin heavy chain genes are shared between at least two species, which became only obvious after having analysed genes from 22 species [9]. It is highly unlikely that introns would have been introduced at the exact same position with exact same reading frame independently in species whose last common ancester had been millions of years ago (600 myr in case of the arthropods). Data from further arthropods showed, that introns initially found to be shared by only two species are actually shared by more species, and that the last common ancestor of the arthropods must have had even more introns than assumed before (data available from CyMoBase [25]). If data from more species and more protein families would be included, shared introns in genes present in the last common ancestor of the eukaryotes could be identified. For other applications that do not demand strict intron conservation, GenePainter could be extended to allow variable positional flexibility.

Binary data can be very useful to reconstruct phylogenetic relationships and is often created from morphological data [26-28]. We used binary data obtained from protein family inventories (subfamily homolog present or absent) to reconstruct the phylogeny of 21 arthropod species [29]. The resulting phylogeny was in agreement with that derived from protein sequence data. The intron pattern of a protein family (Figure 3) can therefore also be used for phylogenetic tree reconstructions, or for combined data analyses. Depending on the taxonomic distribution of the included sequences, intron pattern data of several protein families need to be included to derive discriminative and meaningful information.

\section{Conclusions}

GenePainter is a tool to analyse the conservation of gene structures of eukaryotic proteins. It aligns the gene structures to the respective protein sequences in a multiple sequence alignment. Gene structure conservation can be displayed in a binary format (exons and introns) 


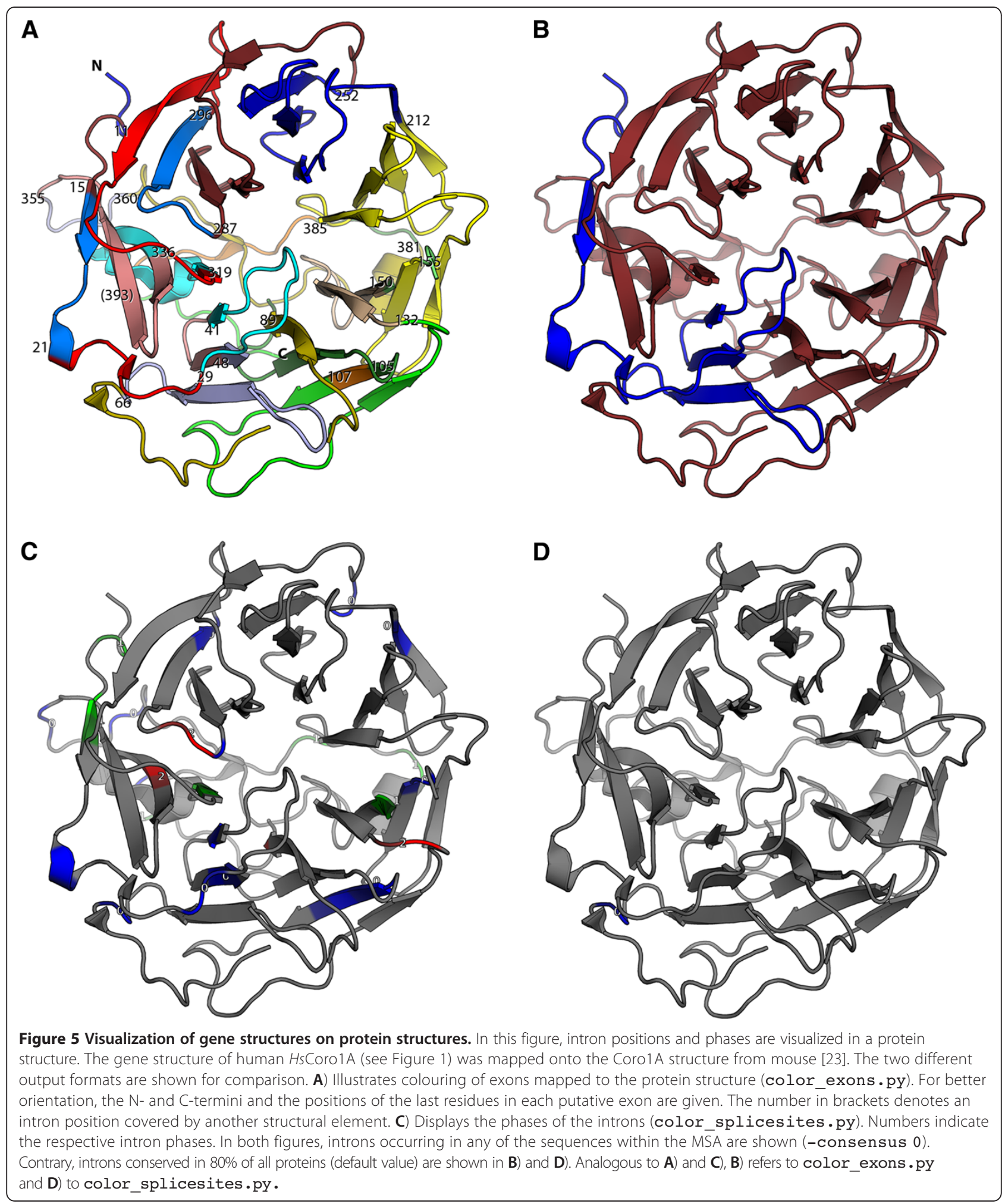


and based on the nucleotide sequences. GenePainter can map gene structure conservation on protein structures and provides scripts for visualization in PyMol. Therefore, GenePainter will be a valuable tool for gene structure guided improvements of multiple sequence alignments and for phylogenetic analyses including or focusing on the conservation of intron positions within eukaryotic genes.

\section{Availability and requirements \\ Project name: GenePainter}

Project home page: http://www.motorprotein.de/ genepainter.html

Operating system: Platform independent

Programming languages: Ruby

Software requirements: Ruby version 1.9.2 or higher

License: GenePainter can be downloaded and used under a GNU General Public License.

Any restrictions to use by non-academics: Using GenePainter by non-academics requires permission.

\section{Additional files}

Additional file 1: This file contains the GenePainter software (gene painter.rb), a README file for installation instructions, the GenePainter documentation, and a script to reconstruct genes via the web service of WebScipio (gene scan.rb). It also includes example data (MSA, gene and protein structures) used to create the figures. This file is also available from the project homepage.

Additional file 2: This file contains a benchmark test of GenePainter on coronin (552 sequences, 1144 alignment positions), dynactin 1 (207 sequences, 2112 alignment positions), dynactin 3 (213 sequences, 428 alignment positions), Wiskott Aldrich Syndrome protein (229 sequences, 2051 alignment positions), and myosin heavy chain genes (2640 sequences, 9214 alignment positions). All sequences and gene structures can be found at CyMoBase (http://www.cymobase.org).

Competing interests

The authors declare that they have no competing interests.

\section{Authors' contributions}

$\mathrm{MK}, \mathrm{FO}$, and SW designed the project and set the requirements of the tool. $\mathrm{BH}$ and $\mathrm{FO}$ wrote the source code. SM added the mapping of the gene structure patterns to PDB files. MK, BH and SM wrote the manuscript. All authors read and approved the final manuscript.

\section{Acknowledgements}

We would like to thank Klas Hatje, Oliver Keller, Malte Hübner, and other members of the Kollmar and Waack groups for helpful suggestions and discussions. MK has been funded by grant KO 2251/6-1 and SW by grant WA 766/6-1 of the Deutsche Forschungsgemeinschaft. This work was partly supported by the Göttingen Graduate School of Neurosciences and Molecular Biosciences (DFG Grant GSC 226/1).

\section{Author details}

'Department of NMR-based Structural Biology, Max-Planck-Institute for Biophysical Chemistry, Am Fassberg 11, Göttingen 37077, Germany. ${ }^{2}$ Institute of Computer Science, University of Göttingen, Goldschmidtstr. 7, Göttingen 37077, Germany.

Received: 10 September 2012 Accepted: 24 February 2013 Published: 4 March 2013

\section{References}

1. Nixon JEJ, Wang A, Morrison HG, McArthur AG, Sogin ML, Loftus BJ, Samuelson J: A spliceosomal intron in Giardia lamblia. Proc Natl Acad Sci USA 2002, 99:3701-3705.

2. Vaňáčová Š, Yan W, Carlton JM, Johnson PJ: Spliceosomal introns in the deep-branching eukaryote Trichomonas vaginalis. Proc Natl Acad Sci USA 2005, 102:4430-4435.

3. Koonin EV: The origin of introns and their role in eukaryogenesis: a compromise solution to the introns-early versus introns-late debate? Biol Direct 2006, 1:22.

4. Carmel L, Wolf YI, Rogozin IB, Koonin EV: Three distinct modes of intron dynamics in the evolution of eukaryotes. Genome Res 2007, 17:1034-1044.

5. De Roos AD: Conserved intron positions in ancient protein modules. Biol Direct 2007, 2:7.

6. Lynch M: Intron evolution as a population-genetic process. Proc Natl Acad Sci USA 2002, 99:6118-6123.

7. Hsieh SJ, Lin CY, Liu NH, Chow WY, Tang CY: GeneAlign: a coding exon prediction tool based on phylogenetical comparisons. Nucleic Acids Res 2006, 34:W280-W284.

8. Csürös M, Holey JA, Rogozin IB: In search of lost introns. Bioinformatics 2007, 23:i87-i96

9. Odronitz F, Kollmar M: Comparative genomic analysis of the arthropod muscle myosin heavy chain genes allows ancestral gene reconstruction and reveals a new type of "partially" processed pseudogene. BMC Mol Biol 2008, 9:21

10. Pavesi G, Zambelli F, Caggese C, Pesole G: Exalign: a new method for comparative analysis of exon-intron gene structures. Nucleic Acids Res 2008, 36:e47-e47.

11. Wilkerson MD, Ru Y, Brendel VP: Common introns within orthologous genes: software and application to plants. Brief Bioinform 2009, 10:631-644

12. Fawal N, Savelli B, Dunand C, Mathé C: GECA: a fast tool for gene evolution and conservation analysis in eukaryotic protein families. Bioinformatics 2012, 28:1398-1399.

13. Csürös M: Malin: maximum likelihood analysis of intron evolution in eukaryotes. Bioinformatics 2008, 24:1538-1539.

14. Fedorov A, Merican AF, Gilbert W: Large-scale comparison of intron positions among animal, plant, and fungal genes. Proc Natl Acad Sci USA 2002, 99:16128-16133

15. Rogozin IB, Wolf YI, Sorokin AV, Mirkin BG, Koonin EV: Remarkable Interkingdom Conservation of Intron Positions and Massive, LineageSpecific Intron Loss and Gain in Eukaryotic Evolution. Curr Biol 2003, 13:1512-1517.

16. Vivek G, Tan TW, Ranganathan S: XdomView: protein domain and exon position visualization. Bioinformatics 2003, 19:159-160.

17. Keller O, Odronitz F, Stanke M, Kollmar M, Waack S: Scipio: using protein sequences to determine the precise exon/intron structures of genes and their orthologs in closely related species. BMC Bioinformatics 2008, 9:278.

18. Odronitz F, Pillmann H, Keller O, Waack S, Kollmar M: WebScipio: an online tool for the determination of gene structures using protein sequences. BMC Genomics 2008, 9:422.

19. Hatje K, Keller O, Hammesfahr B, Pillmann H, Waack S, Kollmar M: Crossspecies protein sequence and gene structure prediction with fine-tuned Webscipio 2.0 and Scipio. BMC Res Notes 2011, 4:265.

20. The PYMOL Molecular Graphics System. Schrödinger, LLC http://www.pymol.org

21. Needleman SB, Wunsch CD: A general method applicable to the search for similarities in the amino acid sequence of two proteins. $J \mathrm{Mol} B \mathrm{BiO}$ 1970, 48:443-453.

22. Eckert C, Hammesfahr B, Kollmar M: A holistic phylogeny of the coronin gene family reveals an ancient origin of the tandem-coronin, defines a new subfamily, and predicts protein function. BMC Evol Biol 2011, 11:268.

23. Appleton BA, Wu P, Wiesmann C: The crystal structure of murine coronin1: a regulator of actin cytoskeletal dynamics in lymphocytes. Structure 2006, 14:87-96.

24. GFF - GMOD. http://gmod.org/wiki/GFF.

25. CyMoBase - a database for cytoskeletal and motor proteins. http://www. cymobase.org.

26. Endress PK, Doyle JA: Reconstructing the ancestral angiosperm flower and its initial specializations. Am J Bot 2009, 96:22-66. 
27. Wiens JJ: Paleontology, genomics, and combined-data phylogenetics: can molecular data improve phylogeny estimation for fossil taxa? Syst Biol 2009, 58:87-99.

28. Werneburg I, Sánchez-Villagra MR: Timing of organogenesis support basal position of turtles in the amniote tree of life. BMC Evol Biol 2009, 9:82.

29. Odronitz F, Becker S, Kollmar M: Reconstructing the phylogeny of 21 completely sequenced arthropod species based on their motor proteins. BMC Genomics 2009, 10:173.

doi:10.1186/1471-2105-14-77

Cite this article as: Hammesfahr et al:: GenePainter: a fast tool for aligning gene structures of eukaryotic protein families, visualizing the alignments and mapping gene structures onto protein structures. $B M C$ Bioinformatics 2013 14:77.

\section{Submit your next manuscript to BioMed Central} and take full advantage of:

- Convenient online submission

- Thorough peer review

- No space constraints or color figure charges

- Immediate publication on acceptance

- Inclusion in PubMed, CAS, Scopus and Google Scholar

- Research which is freely available for redistribution 